\title{
Stereoselective syntheses of aminocyclopentitols: a norbornyl approach
}

\author{
Goverdhan Mehta* and Narinder Mohal \\ Department of Organic Chemistry, Indian Institute of Science, Bangalore 560 012, India
}

\begin{abstract}
Synthesis of some novel aminocyclopentitol analogues has been achieved through a functionally embellished cyclopentanoid derivative of well-defined stereochemical disposition, obtained via a Grob-type fragmentation sequence executed within the norbornyl framework. The glycosidase inhibition studies of the new aminocyclopentitols indicate them to be weak inhibitors.
\end{abstract}

Recent years have witnessed a great deal of interest in the synthesis of polyhydroxylated aminocyclopentanes (aminocyclopentitols), which either exist as such in nature or constitute important sub-structures among several complex natural products, and display a wide range of biological activity profiles. ${ }^{1}$ Among the well known examples of this class of molecules of contemporary interest are glycosidase inhibitors mannostatin A 1, trehazolin 2 and their synthetic analogues such as Merrel Dow's cyclopentylamine $\mathbf{3}$ and recently disclosed aminocyclopentitols $\mathbf{4 a}$ and $\mathbf{4 b}$, and carbocyclic nucleosides such as neplanocin A 5, aristeromycin $\mathbf{6}$ and their analogues. ${ }^{1-3}$ In the context of glycosidase inhibition, these compounds can be considered as structural analogues of carbohydrates containing a basic nitrogen function at the anomeric center in the protonated form. Generally, their mode of action is presumed to proceed via mimicking of the glycopyranosyl cation in the transition state of the glycosidase catalyzed reaction. ${ }^{4}$ Besides 1-6, several aminocyclopentitol based glycosidase inhibitors, having gluco-, manno-, galactoand fuco-stereochemical patterns have been synthesized and evaluated against their corresponding glycosidases in the recent past. ${ }^{1-3}$ However, glycosidase inhibition by aminocyclopentitols as a function of their structural and stereochemical features still remains to be fully understood $^{2 \mathrm{~b}-\mathrm{f}}$ and, therefore, the synthesis of newer analogues could provide not only a better understanding of glycosidase functioning but also lead to more active inhibitors, which can be used for the treatment of carbohydrate metabolism related disorders. ${ }^{4}$

In connection with our ongoing project on the synthesis of carbasugars and azasugars, ${ }^{5}$ we have recently dis-<smiles>C[C@H]1[C@H](O)[C@H](O)[C@@H](O)[C@@H]1N</smiles><smiles>[R]C[C@@H]1[C@H](O)[C@H](O)[C@@H](O)[C@@H]1N</smiles>

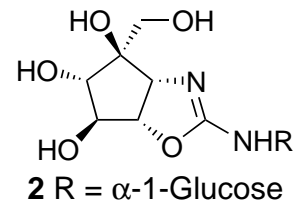<smiles>Nc1ncnc2c1ncn2[C@@H]1C=C(CO)[C@@H](O)[C@H]1O</smiles><smiles>CN[C@@H]1[C@@H](CO)[C@H](O)[C@@H](O)[C@@H]1O</smiles><smiles>Nc1ncnc2c1ncn2[C@@H]1C[C@H](CO)[C@@H](O)[C@H]1O</smiles>

Keywords: aminocyclopentitol; glycosidase inhibitor; carbocyclic nucleosides.

* Corresponding author. 

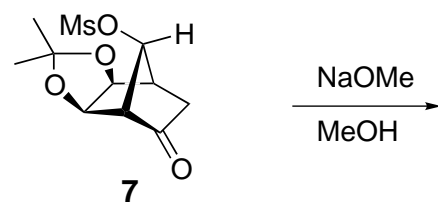

7

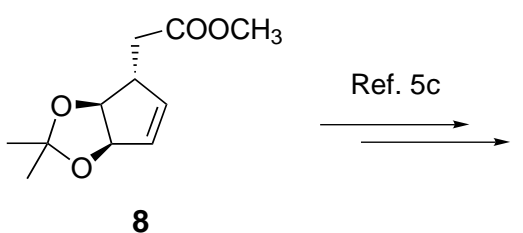

8

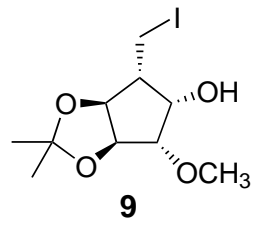

Scheme 1.

closed a stereoselective synthesis of the densely functionalized cyclopentanoid framework 9 through further elaboration of the cyclopentene ester $\mathbf{8}$, obtained in a Grob-type fragmentation sequence orchestrated within the norbornyl framework 7, Scheme $1 .{ }^{5 \mathrm{c}}$ Herein, we report the transformation of cyclopentanoid framework 9 to new aminocyclopentitols having the altrose configuration and to an analogue of the carbocyclic nucleoside neplanocin $\mathrm{A}$, in which the amino and hydroxy functionalities are transposed, and evaluate their glycosidase inhibition activity.

The free hydroxy group in $\mathbf{9}$ was first activated as a leaving group in the form of the mesylate functionality and then the iodide was subjected to reductive dehalogenation with $\mathrm{NaBH}_{3} \mathrm{CN}$ to furnish the methyl compound $10^{6}$ (Scheme 2). In order to set up the requisite amine functionality, the mesylate group in $\mathbf{1 0}$ was displaced using $\mathrm{NaN}_{3}$ as a nucleophile to afford $11 .{ }^{6}$ Catalytic hydrogenation of the azide group in 11 provided an access to the free amine, which was purified and characterized as its acetamide derivative $12 .{ }^{6}$ Acid catalyzed deprotection of the amide as well as the acetonide group in $\mathbf{1 2}$ was effected smoothly to furnish the desired amine, a stereoisomer of the potent inhibitor $\mathbf{4 b},{ }^{2 \mathrm{f}}$ as its hydrochloride salt $\mathbf{1 3}^{6}$ (Scheme 2). In a related sequence, the mesylate $\mathbf{1 4}$ derived from $\mathbf{9}$ on peracid mediated oxidation of the iodomethyl functionality was directly transformed to the hydroxy compound $15 .{ }^{6}$ Alternately, 15 could also be accessed from
14 via dehydroiodination to the exocyclic olefin and hydroboration-oxidation. However, the yield and stereoselectivity in this sequence was rather modest. Further, the mesylate group in $\mathbf{1 5}$ was smoothly displaced by the azide anion to yield 16, which was further transformed to $17,{ }^{6}$ closely related to the powerful inhibitor $\mathbf{4 a},{ }^{2 \mathrm{e}}$ in a two-step sequence involving azide group reduction and acid catalyzed deprotection of the acetonide moiety see (Scheme 2).

Syntheses of novel amino cyclopentitols with an amino group in the side-chain and the carbocyclic nucleoside analogue of neplanocin A 5 in which the purine and hydroxy group positions on the cyclopentitol moiety are swapped was attempted next as outlined in Scheme 3. Sodium azide mediated displacement of the iodomethyl group and concomitant elimination of the mesylate group in $\mathbf{1 4}$ led directly to $\mathbf{1 8}$ with the requisite disposition of the double bond (cf. 5). Reduction of the azide group in $\mathbf{1 8}$ under carefully crafted conditions generated the aminomethyl derivative $19,{ }^{6}$ which upon deprotection of the acetonide protective group yielded the novel cyclopentitol with a primary amino group $\mathbf{2 0}$, as its hydrochloride salt. In a related sequence, sodium acetate mediated displacement of the iodo functionality and concomitant elimination of the mesylate group in 14 furnished 21, which upon alkaline hydrolysis provided access to $22 .{ }^{6}$ To transform 22 into the carbocyclic nucleoside analogue, the adenine moiety was attached to it under Mitsunobu conditions in a regiose-
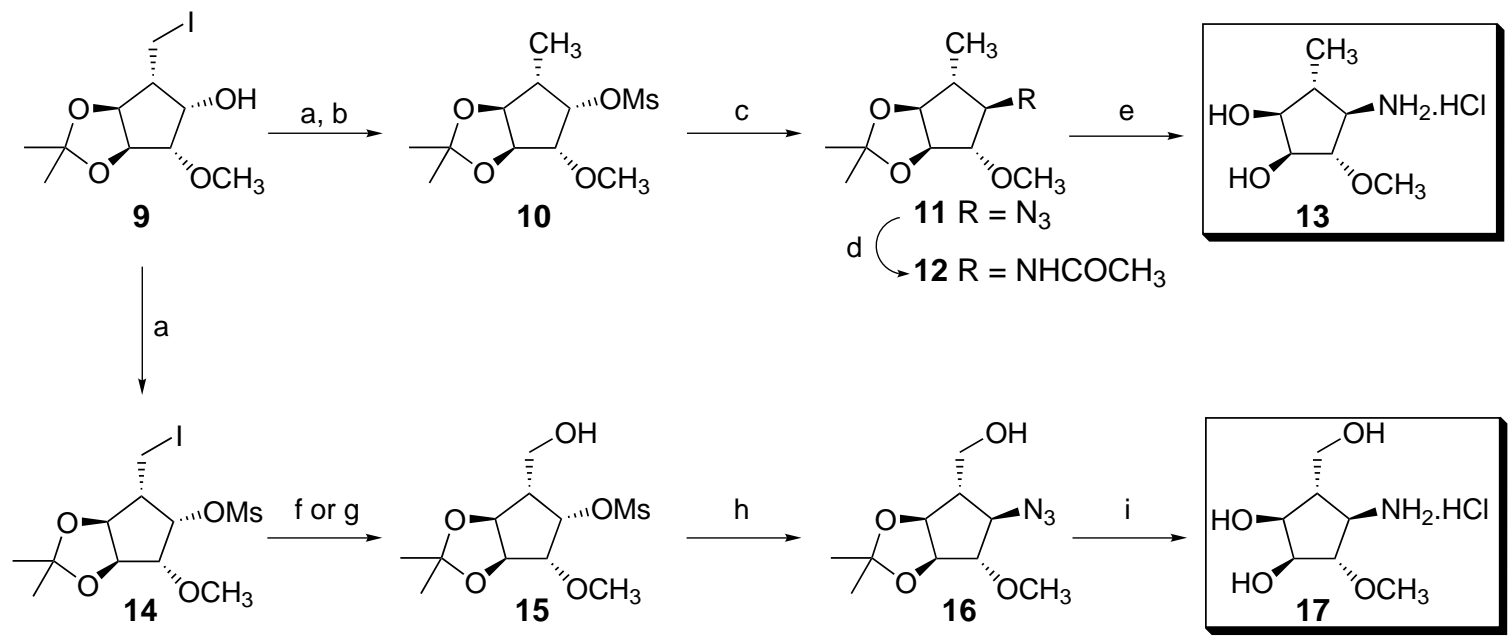

Scheme 2. Reagents and conditions: (a) $\mathrm{MsCl}, \mathrm{Et}_{3} \mathrm{~N}, \mathrm{DCM}-10$ to $0^{\circ} \mathrm{C}, 92 \%$ (b) $\mathrm{NaBH}_{3} \mathrm{CN}, \mathrm{HMPA}, 100^{\circ} \mathrm{C}, 1 \mathrm{~h}, 55 \%$; (c) $\mathrm{NaN}$, DMF, $60^{\circ} \mathrm{C}, 2 \mathrm{~h}, 67 \%$; (d) Lindlar's catalyst, $\mathrm{H}_{2}$, EtOH, $1 \mathrm{~h}$; Ac $\mathrm{Ac}_{2} \mathrm{O}$, DMAP, DCM, 45 min, 82\% for two-steps; (e) 5\% HCl, $\mathrm{Et}_{2} \mathrm{O}-\mathrm{H}_{2} \mathrm{O}(1: 9), 70^{\circ} \mathrm{C}$, >90\%; (f) MCPBA, DCM, $0^{\circ} \mathrm{C}, 5 \mathrm{~h}, 76 \%$; (g) DBU, $\mathrm{CH}_{3} \mathrm{CN}$, overnight, $92 \% ; \mathrm{BH}_{3} \cdot \mathrm{THF}^{\circ}-10$ to rt, overnight, then $30 \% \mathrm{H}_{2} \mathrm{O}_{2}, 3 \mathrm{M} \mathrm{NaOH}, 0^{\circ} \mathrm{C}, 2 \mathrm{~h}, 58 \%$; (h) same as (c), 78\%; (i) Lindlar's catalyst, $\mathrm{H}_{2}, \mathrm{EtOH}, 2 \mathrm{~h} ; 5 \% \mathrm{HCl}$, $\mathrm{Et}_{2} \mathrm{O}-\mathrm{H}_{2} \mathrm{O}(1: 4),>90 \%$. 

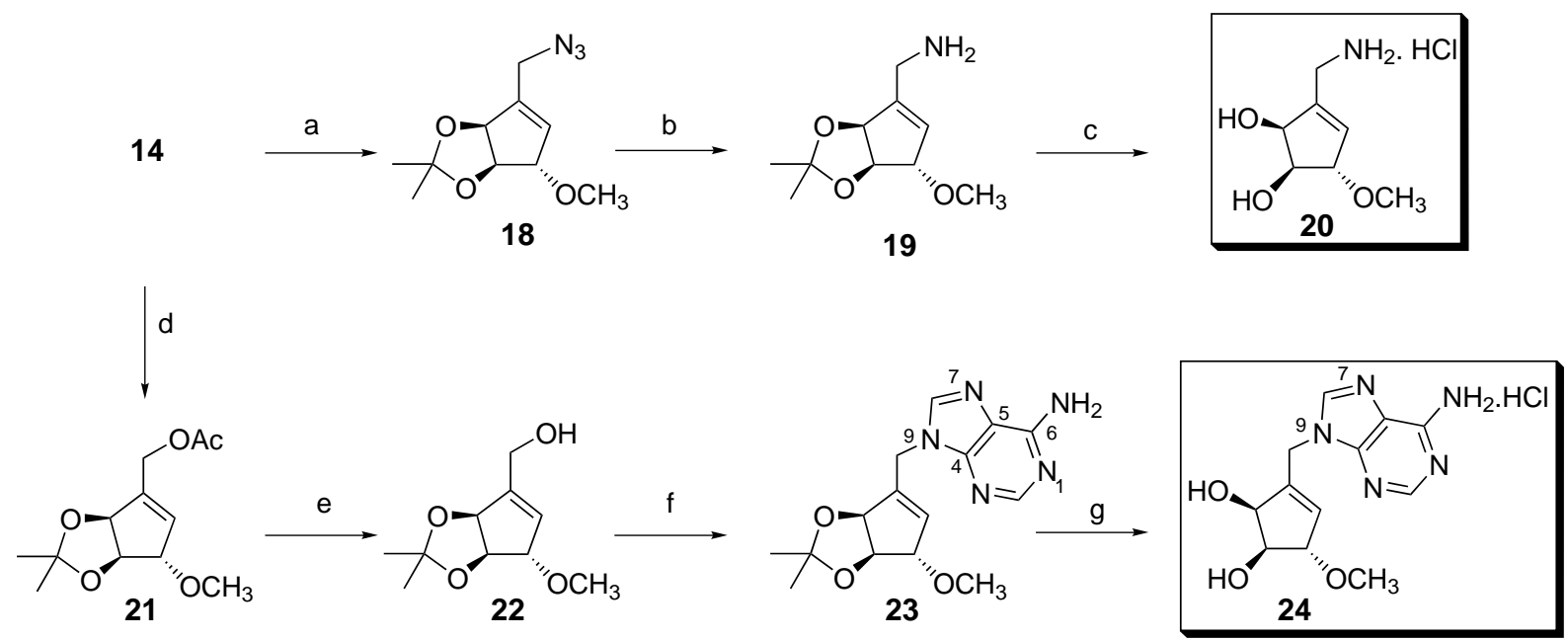

Scheme 3. Reagents and conditions: (a) $\mathrm{NaN}_{3}$, DMF, $60^{\circ} \mathrm{C}, 2 \mathrm{~h}, 67 \%$; (b) $\mathrm{PPh}_{3}$, aq. $\mathrm{NH}_{3}-\mathrm{Py}(1: 1)$, overnight, $93 \%$; (c) $5 \% \mathrm{HCl}$, $\mathrm{Et}_{2} \mathrm{O}-\mathrm{H}_{2} \mathrm{O}$ (1:9), rt, >90\%; (d) NaOAc, DMF, $100^{\circ} \mathrm{C}, 2$ h, 77\%; (e) KOH, MeOH, rt, 2 h, 68\%; (f) DEAD, adenine, 1,4-dioxan, $\mathrm{rt}, 36 \mathrm{~h}, 83 \%$; (g) same as (c), quant.

lective manner to deliver $23 .{ }^{6}$ Acid catalyzed deprotection of the acetonide moiety in $\mathbf{2 3}$ led directly to the formation of 24. ${ }^{6}$ The ${ }^{1} \mathrm{H}$ and ${ }^{13} \mathrm{C}$ NMR spectra of both 23 and 24 clearly indicated the N9 substituted structures rather than the N7 substituted structures ${ }^{7}$. It is to be noted that structural analogues of carbocyclic nucleosides have evoked a great deal of attention ${ }^{1 \mathrm{~b}}$ as anti-viral and anti-HIV agents and to our knowledge $\mathbf{2 4}$ represents a new variation.

Glycosidase inhibitory activity of aminocyclopentitol derivatives 13 and $\mathbf{1 7}$ having the altrose configuration, and of the carbocyclic nucleoside analogue $\mathbf{2 4}$ and the related aminocyclopentene derivative $\mathbf{2 0}$ was evaluated against $\alpha$-glucosidase (yeast), $\beta$-glucosidase (sweet almond), $\alpha$-galactosidase (green coffee beans) and $\beta$ galactosidase (E. coli) using their corresponding orthoor para-nitrophenyl-glycopyranosides as substrates at optimum $\mathrm{pH}$ and temperature of each enzyme. While the methyl derivative $\mathbf{1 3}$ was found to be a weak anomer selective and competitive inhibitor of $\alpha$-glucosidase $\left(K_{\mathrm{i}}=503 \mu \mathrm{M}\right)$, the hydroxymethyl derivative $\mathbf{1 7}$ turned out to be an even weaker inhibitor with only $13 \%$ inhibition at $1 \mathrm{mM}$ concentration. Methyl substituted aminocyclopentitol $\mathbf{1 3}$ showed a non-selective inhibition against $\alpha$ - and $\beta$-galctosidases with 30 and $40 \%$ inhibition, respectively, at $1 \mathrm{mM}$ concentration whereas there was no observable inhibition by $\mathbf{1 7}$ in the case of other glycosidases. This marked decrease in the inhibitory activity of $\mathbf{1 3}$ and $\mathbf{1 7}$, as compared with the closely related diastereomeric aminocyclopentitols reported recently (cf. $\mathbf{4 a}, \mathbf{b}),{ }^{2 \mathrm{~b}-\mathrm{e}}$ could be attributed to the mismatch of configuration with the transition-state of corresponding enzymes. The carbocyclic nucleoside analogue $\mathbf{2 4}$ and the related aminocyclopentene 20 did not show any glycosidase inhibition against the enzymes studied by us, underscoring the need for a wider screen.

In conclusion, we have demonstrated the versatility of readily available cyclopentanoid derivative $9^{5 \mathrm{c}}$ in stereospecific synthesis of two new aminocyclopentitols 13 and 17 with previously unknown stereochemical dispositions. A novel carbocyclic nucleoside analogue $\mathbf{2 4}$ of neplanocin A is also reported. While $\mathbf{1 3}$ was found to be a weak anomer selective inhibitor of $\alpha$-glucosidase, the other cyclopentitols 17, 20, 24 were ineffective as glycosidase inhibitors.

\section{Acknowledgements}

We thank Jawaharlal Nehru Centre for Advanced Scientific Research and CSIR (research fellowship to NM) for supporting this research. We appreciate the help from SIF at IISc, Bangalore, for some of the high field NMR spectra.

\section{References}

1. Recent reviews: (a) Berecibar, A.; Grandjean, C.; Sriwardena, A. Chem. Rev. 1999, 99, 779 and references cited therein; (b) Crimmins, M. T. Tetrahedron 1998, 54, 9229 and references cited therein.

2. Selected references of some of the recent syntheses of aminocyclopentitols and related compounds: (a) Ogawa, S.; Morikawa, T. Eur. J. Org. Chem. 2000, 1759; (b) Blaser, A.; Reymond, J.-L. Org. Lett. 2000, 2, 1733; (c) Blaser, A.; Reymond, J.-L. Synlett. 2000, 817; (d) Boss, O.; Leroy, E.; Blaser, A.; Reymond, J.-L. Org. Lett. 2000, 2, 151; (e) Leroy, E.; Reymond, J.-L. Org. Lett. 1999, 1, 775; (f) Blaser, A.; Reymond, J.-L. Helv. Chim. Acta 1999, 82, 760; (g) Clark, M. A.; Goering, B. K.; Li, J. Ganem, B. J. Org. Chem. 2000, 65, 4058; (h) Johansen, S. K.; Kornø, H. T.; Lundt, I. Synthesis 1999, 171; (i) Dransfield, P. J.; Moutel, S.; Shipman, M.; Sik, V. J. Chem. Soc., Perkin Trans 1 1999, 3349; (j) Kim, K. S.; Park, II, J.; Ding, P. Tetrahedron Lett. 1998, 39, 6471; (k) Marco-Contelles, J.; Gallego, P.; Rodgríues-Fernández, M.; Khiar, N.; Destabel, C.; Bernabé, M.; Martínez-Grau, A.; Chiara, J. L. J. 
Org. Chem. 1997, 62, 7397; (1) Bueno, A. B.; Carreño, M. C.; Gracía Ruano, J. L.; Arrayás, R. G.; Zarzuelo, M. M. J. Org. Chem. 1997, 62, 2139; (m) Nishimura, Y.; Umezawa, Y.; Adachi, H.; Kondo, S.; Takecuchi, T. J. Org. Chem. 1996, 61, 480; (n) Farr, R. A.; Peet, N. P.; Kand, M. S. Tetrahedron Lett. 1990, 31, 7109.

3. For recent synthesis of carbocyclic nucleosides and analogues, see: (a) Hegedus, L. S.; Geisler, L. J. Org. Chem. 2000, 65, 4200; (b) Crimmins, M. T.; King, B. T.; Zuerher, W. J.; Choy, A. L. J. Org. Chem. 2000, 65, 8499; (c) Sharma, P. K.; Nair, V. ARKIVOC 2000, 1, Part 1, 19; (d) Tokoro, Y.; Kobayashi, Y. Chem. Commun. 1999, 807; (e) Roy, A.; Patra, R.; Acari, B.; Mandal, S. B. Synlett 1997, 1237.

4. (a) Zechel, D. L.; Withers, S. J. Acc. Chem. Res. 2000, 33, 11; (b) Heightmann, T. D.; Vasella, A. Angew. Chem., Int. Ed. 1999, 38, 750; (c) Sears, P.; Wong, C.-H. Angew. Chem., Int. Ed. 1999, 38, 2300; (d) Davies, G.; Sinnott, M. L.; Withers, S. G. In Comprehensive Biological Catalysis; Sinnott, M. L., Ed.; Academic Press: London, 1998; p. 119.

5. (a) Mehta, G.; Mohal, N. Tetrahedron Lett. 2000, 41, 5741; (b) Mehta, G.; Mohal, N.; Lakshminath, S. Tetrahedron Lett. 2000, 41, 3505; (c) Mehta, G.; Mohal, N. Tetrahedron Lett. 1999, 40, 5791; (d) Mehta, G.; Mohal, N. Tetrahedron Lett. 1999, 40, 5795.

6. All new compounds reported here were racemic and gave satisfactory spectral data (IR, ${ }^{1} \mathrm{H}$ and ${ }^{13} \mathrm{C} \mathrm{NMR}$, and Mass). Selected spectral data: compound 10: $\delta_{\mathrm{H}}(300$ $\left.\mathrm{MHz}, \mathrm{CDCl}_{3}\right): 5.11(1 \mathrm{H}$, dd as t, $J=4.2 \mathrm{~Hz}), 4.55(1 \mathrm{H}, \mathrm{dd}$, $J=4.5,7.5 \mathrm{~Hz}), 4.37(1 \mathrm{H}, \mathrm{dd}, J=5.7,7.5 \mathrm{~Hz}), 3.78(1 \mathrm{H}$, dd as t, $J=4.2 \mathrm{~Hz}), 3.48\left(3 \mathrm{H}, \mathrm{s},-\mathrm{OCH}_{3}\right), 3.08(3 \mathrm{H}$, s, -OMs $), 2.36-2.23(1 \mathrm{H}, \mathrm{m}), 1.49(3 \mathrm{H}, \mathrm{s}), 1.30(3 \mathrm{H}, \mathrm{s}), 1.20$ $(3 \mathrm{H}, \mathrm{d}, J=8.1 \mathrm{~Hz}) ; \delta_{\mathrm{C}}\left(75 \mathrm{MHz}, \mathrm{CDCl}_{3}\right): 113.39$ (C), $87.15(\mathrm{CH}), 86.58(\mathrm{CH}), 84.0(\mathrm{CH}), 82.82(\mathrm{CH}), 58.09$ $\left(\mathrm{CH}_{3}\right), 41.78\left(\mathrm{CH}_{3}\right), 38.77(\mathrm{CH}), 26.94\left(\mathrm{CH}_{3}\right), 24.44\left(\mathrm{CH}_{3}\right)$, $12.78\left(\mathrm{CH}_{3}\right)$. Compound 13: $\delta_{\mathrm{H}}\left(300 \mathrm{MHz}, \mathrm{D}_{2} \mathrm{O}\right): 3.94$ $(1 \mathrm{H}, \mathrm{d}, J=4.8 \mathrm{~Hz}), 3.59-3.56(2 \mathrm{H}, \mathrm{m}), 3.33(3 \mathrm{H}, \mathrm{s}), 2.90$ $(1 \mathrm{H}, \mathrm{dd}, J=6.3,8.7 \mathrm{~Hz}), 1.95-1.86(1 \mathrm{H}, \mathrm{m}), 1.02(3 \mathrm{H}, \mathrm{d}$, $J=6.9 \mathrm{~Hz}) ; \delta_{\mathrm{C}}\left(100 \mathrm{MHz}, \mathrm{D}_{2} \mathrm{O}\right): 90.81,78.35,76.54$, 61.39, 60.83, 43.71, 17.69. Compound 15: $\delta_{\mathrm{H}}(300 \mathrm{MHz}$, $\left.\mathrm{CDCl}_{3}\right): 5.28(1 \mathrm{H}, \mathrm{t}, J=4.8 \mathrm{~Hz}), 4.51(2 \mathrm{H}, \mathrm{ABq}$ like $\mathrm{m})$, 3.89-3.74 (3H, m), $3.69\left(3 \mathrm{H}, \mathrm{s},-\mathrm{OCH}_{3}\right), 3.1(3 \mathrm{H}, \mathrm{s},-\mathrm{O} M s)$, 2.49-2.47 (1H, m), $1.49\left(3 \mathrm{H}, \mathrm{s},-\mathrm{CH}_{3}\right), 1.27\left(3 \mathrm{H}, \mathrm{s},-\mathrm{CH}_{3}\right)$; $\delta_{\mathrm{C}}\left(75 \mathrm{MHz}, \mathrm{CDCl}_{3}\right): 113.03(\mathrm{C}), 85.60(\mathrm{CH}), 83.0(\mathrm{CH})$, $82.2(\mathrm{CH}), 79.31(\mathrm{CH}), 58.94\left(\mathrm{CH}_{2}\right), 58.17\left(\mathrm{CH}_{3}\right), 49.43$
$(\mathrm{CH}), 38.30\left(\mathrm{CH}_{3}\right), 26.72\left(\mathrm{CH}_{3}\right), 24.18\left(\mathrm{CH}_{3}\right)$. Compound 17: $\delta_{\mathrm{H}}\left(300 \mathrm{MHz}, \mathrm{D}_{2} \mathrm{O}\right): 3.99(1 \mathrm{H}$, dd as t, $J=5 \mathrm{~Hz}), 3.84$ $(1 \mathrm{H}$, dd as t, $J=5.4 \mathrm{~Hz}), 3.77-3.74(2 \mathrm{H}, \mathrm{m}), 3.68-3.64$ $(1 \mathrm{H}, \mathrm{m}), 3.45\left(3 \mathrm{H}, \mathrm{s},-\mathrm{OCH}_{3}\right), 3.28-3.26(1 \mathrm{H}, \mathrm{m}), 2.17-2.11$ $(1 \mathrm{H}, \mathrm{m}) ; \delta_{\mathrm{C}}\left(75 \mathrm{MHz}, \mathrm{D}_{2} \mathrm{O}\right): 87.50(\mathrm{CH}), 74.60(\mathrm{CH})$, $71.68(\mathrm{CH}), 60.98\left(\mathrm{CH}_{2}\right), 58.67\left(\mathrm{CH}_{3}\right), 54.91(\mathrm{CH}), 48.80$ $(\mathrm{CH})$. Compound 19: $\delta_{\mathrm{H}}\left(300 \mathrm{MHz}, \mathrm{CDCl}_{3}\right): 5.84-5.83$ $(1 \mathrm{H}$, br s), $5.13(1 \mathrm{H}, \mathrm{d}, J=6.0 \mathrm{~Hz}), 4.58(1 \mathrm{H}, \mathrm{d}, J=6.0$ $\mathrm{Hz}), 4.30-4.29(1 \mathrm{H}, \mathrm{m}), 3.97(2 \mathrm{H}, \mathrm{ABq}, J=15.3 \mathrm{~Hz}), 3.43$ $(3 \mathrm{H}, \mathrm{s}), 1.40(3 \mathrm{H}, \mathrm{s}), 1.36(3 \mathrm{H}, \mathrm{s}) ; \delta_{\mathrm{C}}\left(75 \mathrm{MHz} \mathrm{CDCl}_{3}\right)$ : $151.13(\mathrm{C}), 124.57(\mathrm{CH}), 111(\mathrm{C}), 89.04(\mathrm{CH}), 84.03(\mathrm{CH})$, $83.31(\mathrm{CH}), 56.90\left(\mathrm{CH}_{3}\right), 49.76\left(\mathrm{CH}_{2}\right), 27.29\left(\mathrm{CH}_{3}\right), 25.74$ $\left(\mathrm{CH}_{3}\right)$. Compound 20: $\delta_{\mathrm{H}}\left(300 \mathrm{MHz}, \mathrm{D}_{2} \mathrm{O}\right): 5.8(1 \mathrm{H}$, br s), $4.47(1 \mathrm{H}, \mathrm{d}, J=6.0 \mathrm{~Hz}), 4.30-4.29(1 \mathrm{H}, \mathrm{m}), 3.94(1 \mathrm{H}, \mathrm{dd}$, $J=4.5,5.7 \mathrm{~Hz}), 3.64\left(2 \mathrm{H}\right.$, br s), $3.33(3 \mathrm{H}, \mathrm{s}) ; \delta_{\mathrm{C}}(75 \mathrm{MHz}$, $\left.\mathrm{D}_{2} \mathrm{O}\right): 140.72(\mathrm{C}), 130.89(\mathrm{CH}), 89.10(\mathrm{CH}), 76.26(\mathrm{CH})$, $74.40(\mathrm{CH}), 57.40\left(\mathrm{CH}_{3}\right), 38.43\left(\mathrm{CH}_{2}\right)$. Compound 22: $\delta_{\mathrm{H}}$ $\left(300 \mathrm{MHz}, \mathrm{CDCl}_{3}\right): 5.77(1 \mathrm{H}$, br s), $5.16(1 \mathrm{H}, \mathrm{d}, J=5.7$ $\mathrm{Hz}), 4.57(1 \mathrm{H}, \mathrm{d}, J=5.7 \mathrm{~Hz}), 4.44 .21(3 \mathrm{H}, \mathrm{m}), 3.43(3 \mathrm{H}$, s), $2.61\left(1 \mathrm{H}\right.$, br s, $\mathrm{D}_{2} \mathrm{O}$ exchangeable $), 1.43(3 \mathrm{H}, \mathrm{s}), 1.36$ $(3 \mathrm{H}, \mathrm{s}) ; \delta_{\mathrm{C}}\left(75 \mathrm{MHz}, \mathrm{CDCl}_{3}\right): 148.88(\mathrm{C}), 125.82(\mathrm{CH})$, $112.17(\mathrm{CH}), 89.03(\mathrm{CH}), 83.65(\mathrm{CH}), 83.27(\mathrm{CH}), 60.08$ $\left(\mathrm{CH}_{2}\right), 57.07\left(\mathrm{CH}_{3}\right), 27.27\left(\mathrm{CH}_{3}\right), 25.72\left(\mathrm{CH}_{3}\right)$. Compound 23: $\delta_{\mathrm{H}}\left(300 \mathrm{MHz}, \mathrm{MeOH}-d_{4}\right): \delta 8.17(1 \mathrm{H}, \mathrm{s}), 8.11(1 \mathrm{H}, \mathrm{s})$, $5.78(1 \mathrm{H}$, br s), $5.07(1 \mathrm{H}, \mathrm{d}, J=5.7 \mathrm{~Hz}), 4.99(2 \mathrm{H}$, br s$)$ $4.52(1 \mathrm{H}, \mathrm{d}, J=5.7 \mathrm{~Hz}), 4.20(1 \mathrm{H}, \mathrm{m}), 3.30(3 \mathrm{H}, \mathrm{s}$, $\left.-\mathrm{OCH}_{3}\right), 1.43\left(3 \mathrm{H}, \mathrm{s},-\mathrm{CH}_{3}\right), 1.36\left(3 \mathrm{H}, \mathrm{s},-\mathrm{CH}_{3}\right) ; \delta_{\mathrm{C}}(75$ $\left.\mathrm{MHz}, \mathrm{MeOH}-d_{4}\right): \delta 157.78(\mathrm{C}), 153.78(\mathrm{CH}), 150.71(\mathrm{C})$, $145.63(\mathrm{C}), 143.04(\mathrm{CH}), 129.92(\mathrm{CH}), 119.84(\mathrm{C}), 113.92$ (C), $89.93(\mathrm{CH}), 84.94(\mathrm{CH}), 84.58(\mathrm{CH}), 57.10\left(\mathrm{CH}_{3}\right)$, $42.83\left(\mathrm{CH}_{2}\right), 27.46\left(\mathrm{CH}_{3}\right), 25.93\left(\mathrm{CH}_{3}\right)$. Compound 24: $\delta_{\mathrm{H}}$ $\left(300 \mathrm{MHz}, \mathrm{D}_{2} \mathrm{O}\right): 8.24(1 \mathrm{H}, \mathrm{s}), 8.19(1 \mathrm{H}, \mathrm{s}), 5.56(1 \mathrm{H}$, br s$)$, $5.07(2 \mathrm{H}$, br s), $4.31(1 \mathrm{H}, \mathrm{d}, J=5.4 \mathrm{~Hz}), 4.20-4.18(1 \mathrm{H}$, br s), $3.84(1 \mathrm{H}, \mathrm{dd}, J=4.5,5.7 \mathrm{~Hz}), 3.37\left(3 \mathrm{H}, \mathrm{s},-\mathrm{OCH}_{3}\right) ; \delta_{\mathrm{C}}$ $\left(75 \mathrm{MHz}, \mathrm{D}_{2} \mathrm{O}\right)$ : $150.58(\mathrm{C}), 149.28(\mathrm{C}), 145.71(\mathrm{CH})$, $142.59(\mathrm{C}), 145.21(\mathrm{CH}), 130.64(\mathrm{CH}), 118.86(\mathrm{C}), 89.06$ $(\mathrm{CH}), 77.74(\mathrm{CH}), 77.01(\mathrm{CH}), 57.70\left(\mathrm{CH}_{3}\right), 43.70\left(\mathrm{CH}_{2}\right)$.

7. The characteristic resonance of the $\mathrm{H}_{2}$ and $\mathrm{H}_{8}$ protons and $\mathrm{C}_{5}$ carbon in the ${ }^{1} \mathrm{H}$ and ${ }^{13} \mathrm{C}$ NMR spectra have been used to distinguish between N9 and N7 substitution patterns in the adenine derivatives, see: (a) Gundersen, L.-L.; Bennche, T.; Rise, F.; Gogoll, A.; Undheim, K. Acta Chim. Scand. 1992, 46, 761; (b) Rasmussen, M.; Hope, J. M. Aust. J. Chem. 1982, 35, 525; (c) Chenon, M.-T.; Pugmire, R. J.; Grant, D. M.; Panzica, R. M.; Townsend, L. B. J. Am. Chem. Soc. 1975, 97, 4627. 